\title{
AN EXACTLY SOLVABLE NON-LINEAR BOLTZMANN EQUATION
}

\author{
M.H. ERNST and E.M. HENDRIKS \\ Instituut voor Theoretische Fysica der Rijksuniversiteit Utrecht, The Netherlands
}

Received 29 December 1978

\begin{abstract}
The initial value problem for a model Boltzmann equation of a two dimensional gas with a continuous or discrete energy distribution function and a transition probability $\delta\left(\epsilon-\epsilon^{\prime}\right)$ is solved exactly; $\epsilon$ and $\epsilon^{\prime}$ are the total energies before and after collision.
\end{abstract}

There exist very few models for which the nonlinear Boltzmann equation (BE) can be solved with arbitrary initial conditions. The only one known to us is a model of McKean [1], which admits only velocitie \pm 1 . Recently an exact solution for special initial conditions has been found [2-4] for a number of Maxwell models. Here we solve the BE for a new model with general initial conditions.

Consider the BE for a distribution function $f(v, t)$, isotropic in velocity space and spatially uniform, which reads in a standard notation [5]

$$
\begin{aligned}
\partial_{t} f & =\int \mathrm{d} v_{1} \int \mathrm{d} \Omega g o(g, \Omega)\left[f f_{1}-f^{\prime} f_{1}^{\prime}\right] \\
& =\int \mathrm{d} v_{1} \mathrm{~d} v^{\prime} \mathrm{d} v_{1}^{\prime} W\left(v v_{1} ; v^{\prime} v_{1}^{\prime}\right)\left[f f_{1}-f^{\prime} f_{1}^{\prime}\right] .
\end{aligned}
$$

Here $\sigma$ is the differential scattering cross section, $\Omega$ the direction of scattering, and $g=\left|v-v_{1}\right|$. Primes indicate a dependence on post collisional velocities. Eq. (1a) can also be expressed in $d$-dimensional form with a transition probability $W\left(v v_{1} ; v^{\prime} v_{1}^{\prime}\right)=2^{-d} g^{3-d}$ $\times \sigma(g, \Omega) \delta\left(v+v_{1}-v^{\prime}-v_{1}^{\prime}\right) \delta\left(v^{2}+v_{1}^{2}-v^{\prime 2}-v_{1}^{\prime 2}\right)$. It leads directly to the usual conservation laws and to the H-theorem.

Tjon and $\mathrm{Wu}$ [3] have proposed a class of models with $W=a(\epsilon) \delta\left(\epsilon-\epsilon^{\prime}\right)$ where $a(\epsilon)$ is a function (yet to be chosen) of the total energy $\epsilon=v^{2}+v_{1}^{2}$ of the colliding particles. The basic simplification of these models is that momentum conservation has been dropped. The BE for these models can be reduced to a form similar to eq. (1a) with a collision rate $g \sigma \propto$ $\epsilon^{d-1} a(\epsilon)$. The choice $a(\epsilon)=\epsilon^{1-d}$ leads to an energy independent collision rate, typical for Maxwell models; here we choose $a(\epsilon)=\epsilon^{2-d}$, yielding a collision rate proportional to $\epsilon$. For comparison, the collision rate in a repulsive intermolecular potential $\left(\sim r^{-n}\right)$ is proportional to $\epsilon^{-k}$ with $k=n^{-1}(d-1)-\frac{1}{2}$, where $\epsilon$ is the relative energy, and $n=\infty$ corresponds to hard spheres. For two dimensions this model can be represented most conveniently in terms of the energy distribution function $F(x, t) \propto f(v, t)$ with $x=\frac{1}{2} v^{2}$, normalizations $\int_{0}^{\infty} \mathrm{d} x F(x, t)=\int_{0}^{\infty} \mathrm{d} x x F(x, t)=1$ (conservation laws), and a final maxwellian $F(x, \infty)=\mathrm{e}^{-x}$. It reads

$$
\begin{aligned}
& \partial_{t} F(x)=\int_{x}^{\infty} \mathrm{d} y \int_{0}^{y} \mathrm{~d} y^{\prime}\left[F\left(y-y^{\prime}\right) F\left(y^{\prime}\right)\right. \\
& -F(y-x) F(x)] \\
& =-(x+1) F(x) \\
& \quad+\int_{x}^{\infty} \mathrm{d} y \int_{0}^{y} \mathrm{~d} y^{\prime} F\left(y-y^{\prime}\right) F\left(y^{\prime}\right) .
\end{aligned}
$$

This 2-dimensional BE can be solved for any given initial distribution $F(x, 0)$ in the following manner: The Laplace transform $G(z, t)=\int_{0}^{\infty} \mathrm{d} x \mathrm{e}^{-x z} F(x, t)$ satisfies

$\left(\partial_{t}-\partial_{z}+1\right) G=z^{-1}\left(1-G^{2}\right)$.

This partial differential equation can be reduced to an ordinary first order Riccati differential equation by 
substituting: $z=x ; z+t=y$ and $G(z, t)=g(x, y)$, satisfying $-g^{\prime}(x)+g(x)+x^{-1} g^{2}(x)=x^{-1}$. Later on integration constants will become arbitrary functions of the "hidden" parameter $y=z+t$. For Maxwell-type molecules $[3,4] G(z, t)$ satisfies the second order partial differential equation $\left(\partial_{t}+1\right) \partial_{z} z G=G^{2}$, where only special solutions (similarity solutions) have been found. Our differential equation can be solved by quadrature after the substitution $g(x)=(1+x)^{-1}$ $+u^{-1}(x)$. The result is

$G(z, t)=\frac{\phi(z+t)+(z-1) \mathrm{e}^{-t}}{(1+z) \bar{\phi}(z+t)-\mathrm{e}^{-t}}$,

where the arbitrary function $\phi(y)=\phi(z+t)$ is to be determined from the initial distribution $F(x, 0)$ or its Laplace transform $G(z, 0) \equiv G_{0}(z)$, yielding $\phi(z)$ $=\left[G_{0}(z)+z-1\right] \cdot\left[(1+z) G_{0}(z)-1\right]^{-1}$. A final Laplace inversion yields the exact solution $F(x, t)$ $=(2 \pi \mathrm{i})^{-1} \int_{-\mathrm{i} \infty}^{\mathrm{i} \infty} \mathrm{d} z \mathrm{e}^{x z} G(z, t)$.

Comments. (i) $G_{0}(z)$ is of course analytic for $\mathrm{Re}$ $z>0$. The same should hold for $G(z, t)$ for all $t$, as it is the Laplace transform of a distribution function. Furthermore, $F(x, t)$ should be positive for all $t$. We have verified both requirements in a large number of cases, we have not been able to find a counter example, but no general proof has been found. However, one can give many examples of $F(x, 0)$ (not necessarily normalizable, nor positive), such that after a sufficiently long time $F(x, t)$ becomes positive, and its Laplace transform analytic in $\operatorname{Re} z>0$ (see eq. (5)).

(ii) The initial growth of a distribution function with $F(x, 0)=0$ for $x>a$, and $F(a, 0) \neq 0$ can in principle be obtained by expanding the exact solution (4) in powers of $t$. A more direct way is to solve eq. (2) by iteration, starting from the given $F(x, 0)$. The dominant growth for small times is $\propto t$ in the interval $x \leqslant 2 a$, and $\propto t^{n}$ in the interval $(n a,(n+1) a)$ with $n \geqslant 2$. This result also implies that new singularities develop at $x=n a$. More generally, initial singularities in $F(x, 0)$ at $x_{i}(i=1,2, \ldots)$ create new ones at $n_{1} x_{1}$ $+n_{2} x_{2}+\ldots$ with $n_{i} \geqslant 0$. The nature of these new singularities depends on the singularities in $F(x, 0)$, and on the transition probabilities, i.e. on the model considered; e.g. in our model, $F(x, 0)=\delta(x-1)$ develops as dominant singularities: a step $\theta(x-2)$, a kink $(x-3) \theta(x-3),(x-4)^{2} \theta(x-4)$ etc. The type of singularity is weaker for larger $x$-values. (iii) The complete time evolution of singularities can be illustrated best by considering an $F(x, 0)$ containing terms like $\Sigma_{i} a_{i} \delta\left(x-x_{i}\right)$. Its Laplace transform $G_{0}(z)$ behaves like $\Sigma_{i} a_{i} \exp \left(-x_{i} z\right)$, and hence $\left|G_{0}(\mathrm{i} \eta)\right|$ $\simeq$ constant for $\eta \rightarrow \pm \infty$. Eq. (4) implies $G(z, t) \simeq \mathrm{e}^{-t}$ $\times G_{0}(z+t)$ for $\eta=\operatorname{Im} z \rightarrow \pm \infty$ at fixed $t$, and consequently $F(x, t)$ contains contributions $F_{\delta}(x, t)=\Sigma_{i} a_{i}$ $\delta\left(x-x_{i}\right) \exp \left[-\left(x_{i}+1\right) t\right]$. An important point to notice is that an initial $\delta$-peak does not widen, but keeps its shape, while its strength decreases at a rate $\left(x_{i}+1\right)$. At the same time it develops at wide foot (see (ii)). The new singularities decay similarly; in addition the type of singularity may change as time proceeds.

(iv) For a discussion of the decay of the complete initial distribution it is convenient to replace the integration path $(-i \infty,+i \infty)$ in the Laplace inversion formula by closed contours in $\operatorname{Re} z<0$, as can be done in most cases. The arguments are: suppose that $F(x, 0)$ does not contain any $\delta$-peaks, then $\left|G_{0}(\mathrm{i} \eta)\right|$ is smaller than a constant for $\eta=\operatorname{Im} z \rightarrow \pm \infty$, and the same holds for $|G(\mathrm{i} \eta, t)|$ at fixed $t$. If $F(x, t)$ does contain $\delta$-peaks, they should be first subtracted out, and then the same arguments apply. Furthermore, in cases where $G(z, t)$ grows at most like a power of $\zeta$ for $\zeta=\operatorname{Re} z \rightarrow-\infty$, the contours can be closed. In cases where $G(z, t)$ behaves exponentially like $\exp (-\alpha \zeta)$ for $\zeta \rightarrow \pm \infty$, the contours can also be closed but their contributions should be multiplied by a factor $\theta(x-\alpha)$. The final approach towards the maxwellian $F(x, \infty)=\mathrm{e}^{-x}$ can now be discussed in terms of contributions from contours around poles and branch cuts of $G(z, t)$. For large $t$ the dominant (Maxwell) pole in eq. (4) is located at $z_{0} \simeq-1+p_{0}(t)$, with $p_{0}(t) \simeq \mathrm{e}^{-t} / \phi(t-1) \simeq \mathrm{O}\left(\mathrm{e}^{-t}\right)$, since $|\phi(t)|>$ constant for $t>1$. In addition, there may be moving poles at $z_{n}=-t+c_{n}+p_{n}(t)$, where $\phi\left(c_{n}\right)=0$ with $n \geqslant 1$ and $p_{n}(t) \simeq \mathrm{O}\left(\mathrm{e}^{-t}\right)$. Hence, for large $t$ the total contribution from the poles, $F_{p}(x, t)$, becomes

$$
\begin{aligned}
& F_{p}(x, t) \simeq\left[1+\mathrm{O}\left(\mathrm{e}^{-t}\right)\right] \mathrm{e}^{-x} \mathrm{e}^{x p} 0^{(t)} \\
& \quad+\sum_{n} \mathrm{O}\left(\mathrm{e}^{-t}\right) \mathrm{e}^{-x t} \exp \left[x\left(c_{n}+p_{n}(t)\right)\right],
\end{aligned}
$$

where we have only indicated the order of magnitude of the residues. The possible branch points of $G(z, t)$, originating from branch points of $\phi(z)$, are all moving in time. Hence, the contributions from branch cuts are proportional to $\mathrm{e}^{-x t}$ (as are those from the poles), 
and the dominant long time behaviour is given by the first terms in eq. (5), i.e. $F(x, t) / F(x, \infty) \simeq \exp \left(x p_{0}(t)\right)$ with $p_{0}(t)=\mathrm{O}\left(\mathrm{e}^{-t}\right)$. This approach is non-uniform, since for thermal energies, $x \sim 1$, it takes a time $t_{0} \simeq 1$ (in units of mean free times), and for very large $x$ it takes a time $t_{0} \simeq \log x$ before $x p_{0}(t)$ becomes $<1$, i.e. before the Maxwell value has been reached. For similar results, see ref. [2]. The detailed form of the long time behaviour of $p_{0}(t)$ depends on the asymptotics of $\phi(t)$ or $G_{0}(t)$, and, therefore, on the small $x$-behaviour of $F(x, 0)$. This introduces at most a few factors of $t^{-1}$ into $p_{0}(t)$, but does not change qualitatively the dominant dependence of $p_{0}(t) \simeq \mathrm{O}\left(\mathrm{e}^{-t}\right)$. We only note a small tendency of a faster approach to the maxwellian, the closer $F(x, \infty)$ for small $x$.

(v) The linearized BE $\partial_{t} h=\Lambda h$ for $F(x, t)=\mathrm{e}^{-x}$ $\mathrm{X}(1+h(x, t))$ can be obtained in explicit form from eq. (2), and can, of course, be solved exactly as an initial value problem. Of special interest in kinetic theory are the eigenvalues $\lambda$ and the eigenfunctions $h(x, t)$ $=\mathrm{e}^{-\lambda t} h(x)$ of the linearized collision operator $\Lambda$, which is symmetric and negative semi-definite, as one can show by introducing a scalar product $(f, g)=\int_{0}^{\infty} \mathrm{d} x$ $X \cdot e^{-x} f(x) g(x)$. The Hilbert-Enskog canonical form [5] for $\Lambda$ reads in our case $\Lambda h=K h-(x+1) h$, where the Hilbert operator $K h=\int_{0}^{\infty} \mathrm{d} y K(x, y) h(y)$ has the (symmetrized) kernel $\widetilde{K}(x, y)=\exp \left(-\frac{1}{2}(x\right.$ $-y)) K(x, y)=2 \exp \left(-\frac{1}{2}|x-y|\right)-(x+y) \exp \left(-\frac{1}{2}(x\right.$ $+y))$. Unlike e.g. the hard sphere case [5] the Hilbert operator is here non-compact (the first term has actually a continuous spectrum in the interval $(0,8))$. The complete operator $\Lambda$ has a two-fold degenerate eigenvalue $\lambda=0$, with orthogonal eigenfunctions $h_{0}(x)=1$ and $h_{1}(x)=x-1$, and a continuous spectrum $\lambda=\mu$ +1 with $\mu>0$. The eigenfunctions in the orthogonal complement of the null space $\left(h_{0}, h_{1}\right)$ can be obtained directly from the linearized form of eq. (3), and $\operatorname{read} h(x, \mu)=\mathrm{e}^{\mu / 2}\{\delta(x-\mu)+\theta(x-\mu)(x-\mu-2)\}$ with orthogonality relations $\left(h(\mu), h\left(\mu^{\prime}\right)\right)=\delta\left(\mu-\mu^{\prime}\right)$. There are for this model no discrete eigenvalues in the gap $(0,1)$, as follows from the completeness of the set $\left\{h_{0}(x), h_{1}(x), h(x, \mu)\right.$ with $\left.\mu>0\right\}$.

(vi) The moments, $M_{n}(t)=\int_{0}^{\infty} \mathrm{d} x x^{n} F(x, t)=(-)^{n}$ $\times G^{(n)}(0, t)$, where $G^{(n)}$ is the $n$th derivative of $G(z, t)$ with respect to $z$, can also be obtained directly from the exact solution (4). The conservation laws prescribe $M_{0}(t)=M_{1}(t)=1$. By comparing the moments $M_{n}(t)$ for $n \geqslant 2$ with the corresponding mo- ments $M_{n}^{(\mathrm{L})}(t)$, obtained from the linearized BE, one sees that none of them are equal, not even in the leading deviations from $M_{n}(\infty)=M_{n}^{(\mathrm{L})}(\infty)=n$ ! for long times. This is unlike the case of Maxwell molecules, where $M_{n}(t)=M_{n}^{(\mathrm{L})}(t)$ for $n=2,3$.

(vii) An exactly solvable model for a binary mixture ( $i, j=1,2$ labels the components) in two dimensions is found by choosing $W_{i j}\left(v v_{1} ; v^{\prime} v_{1}^{\prime}\right)=m_{i} m_{j} \delta(\epsilon$ $\left.-\epsilon^{\prime}\right)$ where $\epsilon=m_{i} v^{2}+m_{j} v_{1}^{2}$ is the total energy in an $(i, j)$-collision.

(viii) A discrete version of eq. (2), where the distribution function, $F_{n}(t)$, depends on discrete energies $n$, reads $\dot{F}_{n}=\Sigma \delta_{n+m, n^{\prime}+m^{\prime}}\left(F_{n^{\prime}} F_{m^{\prime}}-F_{n} F_{m}\right)$. The sum runs over $m, n^{\prime}, m^{\prime}=0,1,2, \ldots$ This BE obeys the conservation laws $\Sigma_{n} F_{n}=1$ and $\Sigma_{n} n F_{n}=\epsilon$. The equation for the generating function $G(x, t)=\Sigma_{n} x^{n}$ $F_{n}(t)$ (compare eq. (3)) can be solved similarly, and we find the general solution

$G(x, t)=\frac{\phi\left(x \mathrm{e}^{-t}\right)+(\epsilon-\epsilon x-x) \mathrm{e}^{-(\epsilon+1) t}}{\left(\underline{1+\epsilon-\epsilon x) \phi\left(x \mathrm{e}^{-t}\right)-x \mathrm{e}^{-(\epsilon+1) t}}\right.}$,

where the arbitrary function $\phi(x)$ can be determined from the given initial value $G(x, 0)=G_{0}(x)$. Many of the previous comments can be repeated. We only note that the linearized collision operator has a discrete spectrum $\lambda_{k}$ with $\lambda_{k}=0$ for $k=0,1$ and $\lambda_{k}=\epsilon+k$ -1 for $k \geqslant 2$. The eigenfunctions, which will not be quoted here, are orthogonal polynomials, defined on the integers. Finally, a minor modification of the transition probabilities in this discrete version yields yet another solvable BE.

Details on the reported results will be published elsewhere.

It is a pleasure to thank N.G. van Kampen, J. Tjon and I. de Schepper for stimulating discussions and valuable comments.

\section{References}

[1] H.P. McKean Jr., J. Comb. Theory 2 (1967) 358.

[2] A.V. Bobylev, Sov. Phys. Dokl. 20 (1976) 822; M. Krook and T.T. Wu, Phys. Fluids 20 (1977) 1589.

[3] J. Tjon and T.T. Wu, preprint, Univ. of Utrecht (August 1978).

[4] M.H. Ernst, Phys. Lett. 69A (1979) 390.

[5] J.D. Foch and G.W. Ford, Studies in statistical mechanics V, eds. J. de Boer and G.E. Uhlenbeck (North-Holland, Amsterdam, 1970) p. 128. 